\title{
An electrical equivalent circuit to simulate the output power of an AlGaAs/GaAs planar Gunn diode
}

\author{
Mohamed Ismaeel Maricar ${ }^{1}$, Ata Khalid ${ }^{2}$, Geoff Dunn ${ }^{3}$, Steve Greedy ${ }^{1}$, Dave Thomas ${ }^{1}$, D R S \\ Cumming ${ }^{2}$ and $\mathrm{C} \mathrm{H}_{\mathrm{O}} \mathrm{Xley}^{4}$

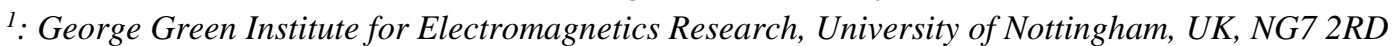 \\ 2: Microsystems Technology Research Group, University of Glasgow, UK, G12 8LT \\ ${ }^{3}$ : School of Natural and Computing Science, University of Aberdeen, UK, AB24 $3 U E$ \\ 4: Independent Academic, Nottingham, UK \\ Email: choxley0@gmail.com
}

\begin{abstract}
The planar Gunn diode offers the potential of microwave, milli-metric and THz based oscillator which can be fabricated as part of a microwave monolithic integrated circuit (mmic). To-date the RF output power has been too low for many applications. This paper looks at a simple electrical equivalent circuit model representation of an aluminium gallium arsenide (AlGaAs) based planar Gunn diode with an active channel length of approximately $4 \mu \mathrm{m}$ and width of $120 \mu \mathrm{m}$. The model indicated a maximum RF output power of $+5 \mathrm{dBm}$ compared with published experimental results of 19dBm for similar diodes.
\end{abstract}

Keywords: GaAs, planar Gunn diode, electrical equivalent circuit model, active channel length, impedance, RF output power.

\section{INTRODUCTION}

The Gunn diode was first demonstrated by J.B Gunn in 1963 using a slab of gallium arsenide ( $\mathrm{GaAs}$ ) sandwiched between two ohmic contacts [1], which led to the successful development and commercial exploitation of the vertical Gunn diode. The vertical Gunn diode configuration was attractive for integrating into waveguide circuits but not suitable for integrating into the new microwave monolithic integrated circuit (mmic) technologies, which emerged in the early ' 80 's. Therefore Gunn diode research/development virtually stopped. However, recently there has been renewed interest in the Gunn diode but in a planar configuration, this configuration makes it attractive for integration into mmic technology opening the door to the potential of miniature, low voltage, low cost integrated microwave, milli-metric and THz rf sources. The planar Gunn diode consists of an active channel, where the narrow length (anode to cathode separation) determines the operating frequency, and the channel width the magnitude of the current. The magnitude of the current will directly influence the RF output power. In 2007 the first aluminium gallium arsenide (AlGaAs) planar Gunn diode operating above $100 \mathrm{GHz}$ with an RF output power of $-43.5 \mathrm{dBm}$ [2-3] was reported. The diode had an active channel length of $1.3 \mu \mathrm{m}$ and a channel width of $120 \mu \mathrm{m}$. Recently published results for a state-of-the-art $\mathrm{In}_{0.53} \mathrm{Ga}_{0.47} \mathrm{As}$ planar Gunn diode with a $1.3 \mu \mathrm{m}$ channel length operated at a fundamental frequency of $164 \mathrm{GHz}$ [4] and with a $0.6 \mu \mathrm{m}$ channel length $307 \mathrm{GHz}$ [5], the output powers were 100 and $28 \mu \mathrm{W}$ respectively. The RF output powers from these devices have been low. This was further emphasised in 2015 with published results for partially on chip matched AlGaAs/GaAs planar Gunn diode operating at $29 \mathrm{GHz}$, where the measured output power was $-19 \mathrm{dBm}[6]$. The low RF output power of the planar Gunn diode, has precluded its use in many applications, and is thought to be due to a number of factors including non-uniform current flow across the channel, resistive parasitics, high junction temperatures and poor match to the diode. The vertical Gunn diode is normally mounted in a waveguide cavity and can therefore be easily tuned using different diode impedance mounting posts and external tuning screws. This gave enormous leeway for matching individual diodes for maximum RF output power. Whereas, the planar Gunn diode and matching circuit will have to be fabricated on chip giving little if any freedom for individual tuning to obtain maximum RF output power. Therefore, to overcome this problem improved on-chip matching of the planar Gunn diode to the external circuit is seen as one way to improve the RF output power.

An oscillator circuit can be thought of as consisting of a negative resistance device, a resonating circuit, and a circuit to match the oscillator to a $50 \mathrm{Ohm}$ load. The first order electrical equivalent circuit model [7] of a Gunn diode is shown in Figure 1 and is represented as a negative conductance $G_{D}$ in parallel with the diode capacitance $C_{D}$. To realise a Gunn oscillator a high $\mathrm{Q}$ inductor $\mathrm{L}$ can be used to form the resonant circuit with the diode capacitance, the resonant frequency is given by $f_{0}=\left(L C_{D}\right)^{0.5}$. The oscillator is then impedance matched to the required load for maximum dissipated RF power.

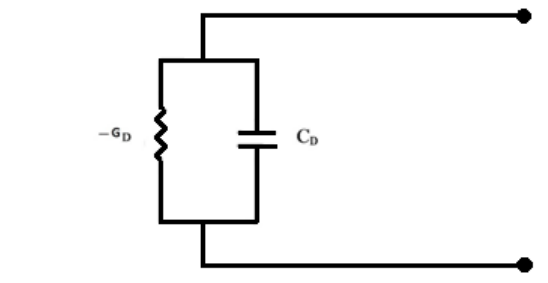

Figure-1 Equivalent circuits model of the planar Gunn diode 
The electrical equivalent circuit model for the planar Gunn diode was derived from experimentally characterising the planar Gunn diode. The negative conductance was derived from pulsed DC IV characteristics, and represented as a nonlinear current generator. The diode capacitance $C_{D}$ was extracted from the small-signal s-parameters. To further improve the model a series parasitic resistance was added to represent the diode anode and cathode contact resistance. For the diode to oscillate the negative resistance will have to be greater than the fixed parasitic resistance.

\section{DERIVATION OF THE SIMPLE ELECTRICAL EQUIVALENT CIRCUIT MODEL}

Planar Gunn diodes were fabricated on $\mathrm{AlGaAs} / \mathrm{GaAs}$ wafer which has already been adequately described in the literature $[6,8]$. Figure-2 shows a SEM image of the planar diode chip with an active channel length of $\approx 4 \mu \mathrm{m}$ and channel width $120 \mu \mathrm{m}$. The diode transit mode oscillation frequency depends on the anode and cathode separations $\left(L_{a c}\right)$ and can be approximated by equation (1)

$$
\left(f=\frac{V_{\text {domain }}}{L_{\text {ac }}}\right)
$$

Where $v_{\text {domain }}$ is the domain velocity. The expression can be refined by subtracting the dead-space from the active length $\mathrm{L}_{\mathrm{as}}$ [9]. The estimated fundamental transit oscillation frequency was approximately $28 \mathrm{GHz}$. To enable RF connection to the planar Gunn diode, it was embedded in a $50 \Omega$ coplanar waveguide (cpw) structure (Figure 2) with a ground source ground (G-S-G) spacing of 40-60-40 $\mu \mathrm{m}$. The diodes were fabricated at the James Watson Nanofabrication Centre (JWNC) at University of Glasgow.

Initially the diodes pulsed IV characteristics (to minimise self-heating) were measured using a semiconductor analyser (Agilent Technologies B1500A), which was connected to a

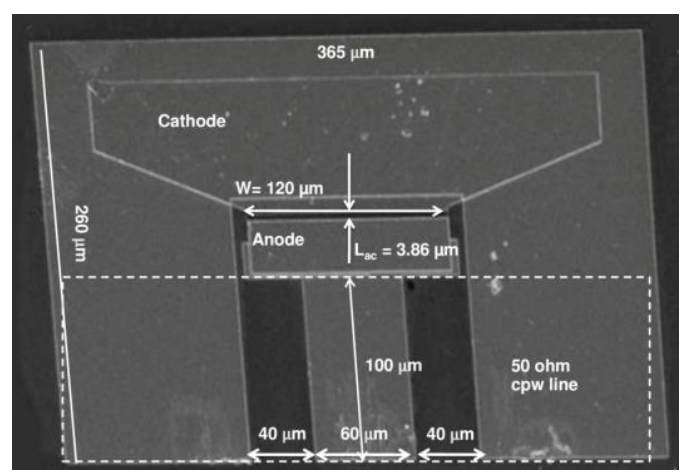

Figure-2 SEM image of $4 \mu \mathrm{m}$ GaAs based planar Gunn diode

Computer controlled automated probe station. The measurements identified a negative differential resistance (NDR) region at a bias voltage of approximately $3.8 \mathrm{~V}$ with a peak current of approximately $91.0 \mathrm{~mA}$. Figure 3 shows the typical measured pulsed IV characteristic.

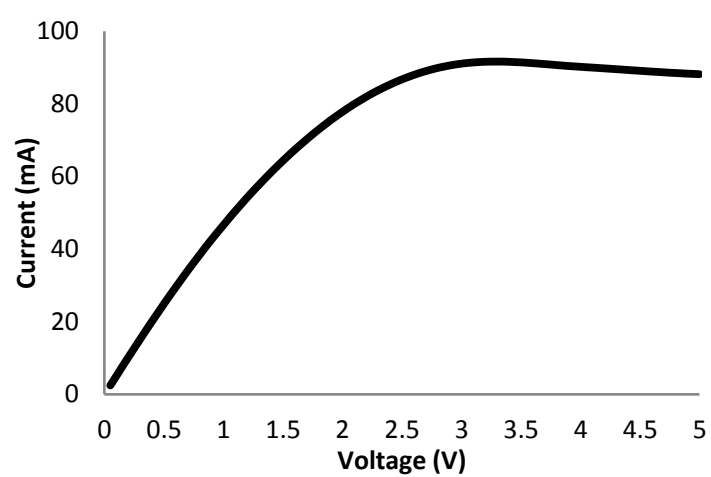

Figure-3 Measured IV characteristics of the a nominal 4 x $120 \mu \mathrm{m}$ AlGaAs based planar Gunn diode

The planar Gunn pulsed IV characteristic was modelled as a non-linear voltage controlled current source (VCCS), using a fifth order polynomial (2) [9].

$I(V)=-7.23 E^{-6} * V^{5}+3.24 E^{-4} * V^{4}-2.31 E^{-3} * V^{3}-$ $2.426 E^{-3} * V^{2}+4.724 E^{-2} * V-3.25 E^{-4}$

The anode and cathode electrode contact parasitic resistance was obtained from the specific resistance of the contacts using transmission line measurement (TLM) structures fabricated on the same wafer as the planar Gunn diodes. The specific contact resistance was extracted as $\approx 0.75 \Omega-\mathrm{m}^{2}$, giving an estimated total contact resistance (anode and cathode) of $3.2 \Omega$.

The planar Gunn diode capacitance was obtained from on wafer small signal s-parameter $\left(s_{11}\right)$ measurements over a frequency range of DC to $110 \mathrm{GHz}$. The diode was biased at different voltage levels ( 0 to $4.1 \mathrm{~V}$ ). The input cpw $50 \Omega$ line was $100 \mu \mathrm{m}$ in length (Figure 2 ) and was de-embedded from the small signal s-parameter measurements, using Keysights Advanced Design Systems (ADS-2009) software. The extracted imaginary part of the s-parameter had a negative reactance and for simplicity was assumed to represent the total capacitance (parasitic and domain [10]) of the Gunn device. The value of the capacitor was found to be approximately $107 \mathrm{fF}$ and to a first order, was almost invariant over a wide frequency and bias voltage range around the NDR region. The derived equivalent circuit model of the planar Gunn diode is shown in Figure-4. It was assumed that junction temperature effects were negligible (preliminary experimental thermal mapping in the channel region indicated $<80^{\circ} \mathrm{C}$ rise in temperature) and the current flow between the electrodes was uniform.

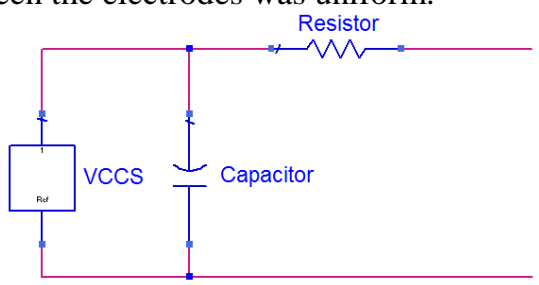

Figure-4 Equivalent circuit model of the planar Gunn diode (ADS model)

The oscillator was designed to operate at its estimated fundamental transit frequency of around $28 \mathrm{GHz}$. To resonate the diode capacitance at $28 \mathrm{GHz}$ an ideal series inductor $0.26 \mathrm{nH}$ was used. The bias was supplied by a low pass filter and a dc block included in the RF line. An electrical 
schematic of the oscillator circuit is shown in Figure 5. The oscillator circuit was simulated using the Advanced Design Systems (ADS -2009) software.

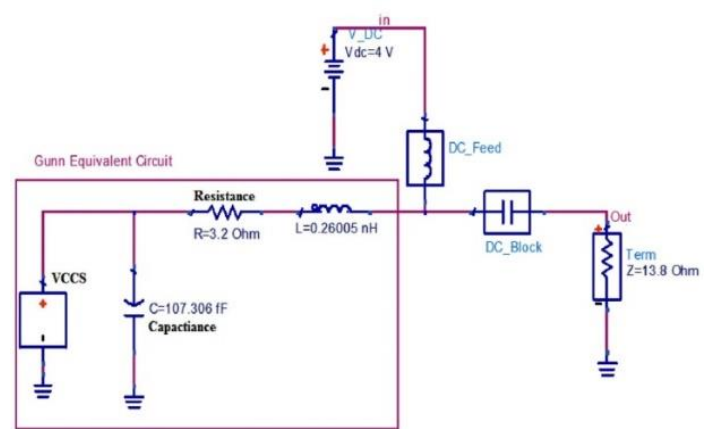

Figure 5 Gunn oscillator circuit

Figure- 6 shows the simulated $s_{11}$ of the planar Gunn diode oscillator represented by the equivalent circuit model in Figure 5. With a bias of $4 \mathrm{~V}$ applied to the Gunn diode the oscillation frequency was $28.2 \mathrm{GHz}$ and increased to $28.5 \mathrm{GHz}$ at $5 \mathrm{~V}$ and disappeared at $5.5 \mathrm{~V}$. Further, if the bias voltage was reduced to below $3.8 \mathrm{~V}$ the transit oscillation disappeared as the diode was no-longer in the NDR region. This behaviour was similar to that expected in experimental measurements.
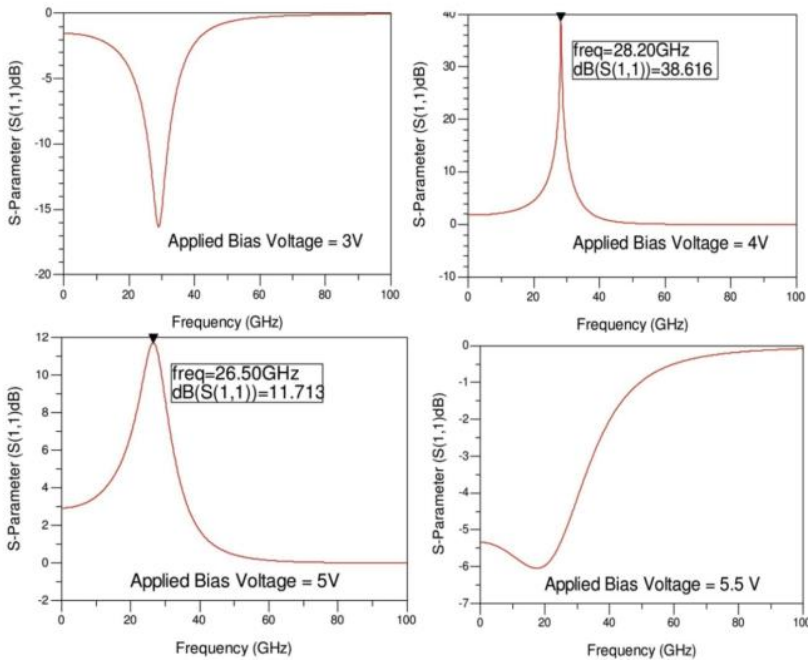

Figure-6 Simulated S-parameter of the $3.8 \mu \mathrm{m}$ planar GaAs based oscillator.

The simulated RF output power is shown in Figure 7, the maximum power was approximately $-0.5 \mathrm{dBm}$ at $4.1 \mathrm{~V}$, which was considerably higher than reported experimental results for similar diodes [6]. The results suggest the oscillator model is too ideal not taking into account all the parasitic losses, non-uniform channel current flow or temperature effects.

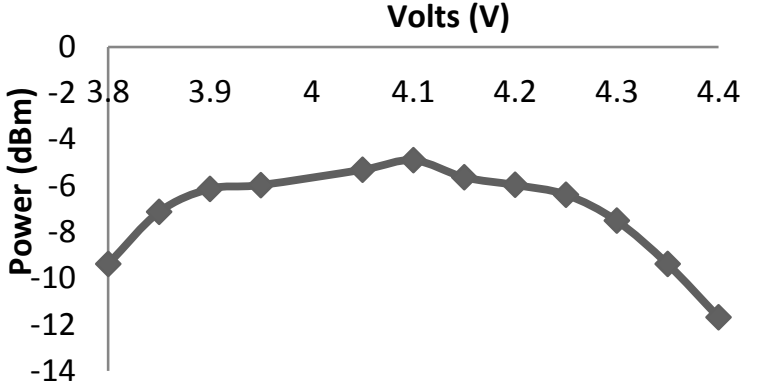

Figure 7 Output power of unmatched Gunn oscillator as a function of bias voltage.

The oscillator was then matched to the $50 \Omega$ load using a lowloss $\lambda / 4$ matching transformer of characteristic impedance $\mathrm{Z}_{0}$, which was inserted between the series inductor and the $50 \Omega$ load. The impedance $\mathrm{Z}_{0}$ was varied to give a maximum power into the load. The maximum power obtained was $+5 \mathrm{dBm}$ when $\mathrm{Z}_{0}$ was approximately $20.0 \Omega$ and the Gunn bias 4.1V, Figure 8 .

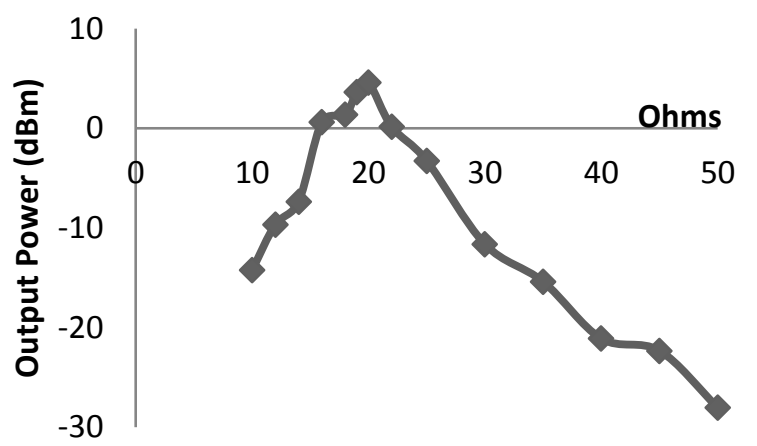

Figure 8 Maximum matched power from Gunn oscillator

The work indicates using the simple electrical equivalent circuit model based on the measurements from an $\mathrm{AlGaAs} / \mathrm{GaAs}$ planar Gunn diode mounted in an ideal resonant oscillator circuit and matched to $50 \Omega$ produces useable output powers of $+5 \mathrm{dBm}$. This figure being an order of 200x greater than the reported experimental output powers from similar diodes. Interestingly, matching the oscillator to $50 \Omega$ improved the power from $-5 \mathrm{dBm}$ to $+5 \mathrm{dBm}$ even if other parasitic effects and non-uniform current flow in the channel were not taken into account. Therefore a major contribution to low output power is impedance matching to $50 \Omega$. The preliminary work in this paper suggests the need for an improved electrical equivalent circuit model to represent the planar Gunn diode in order to design on chip resonant and matching circuits for maximum RF output power. There is little latitude to fine tune the planar Gunn oscillator, unlike the vertical Gunn diode mounted in an oscillator waveguide cavity, which can be fine-tuned using a combination of different diode mounting posts and matching screws. 


\section{Conclusion:}

A planar Gunn diode with an anode to cathode separation of approximately $4 \mu \mathrm{m}$ and a channel width of $120 \mu \mathrm{m}$ was DC and $\mathrm{rf}$ characterised to obtain an electrical equivalent circuit model for the planar Gunn diode. The model assumed a number of simplifications including (i) the total diode capacitance remained constant, (ii) uniform current flow across the channel region and (iii) temperature effects were ignored. The equivalent circuit model was used to design an oscillator working at the natural Gunn transit frequency of approximately $28 \mathrm{GHz}$ and matched to $50 \Omega$. The maximum output power was $+5 \mathrm{dBm}$. This was significantly higher than published experimental rf output powers for similar diodes. The work indicated that matching alone increased the output power from $-5 \mathrm{dBm}$ to $+5 \mathrm{dBm}$. As the planar Gunn diode oscillator cannot be tuned by external screws unlike the vertical Gunn diode in a waveguide cavity there is the need to further develop the electrical equivalent circuit model of the planar Gunn diode. This would enable on chip design of the resonant and matching circuitry, to realise the full potential of the planar Gunn diode as a microwave, millimetric or $\mathrm{THz}$ source.

\section{REFERENCES}

[1] J. B. Gunn, "Microwave oscillations of current in III-V semiconductors," Solid State Commun., vol. 1, no. 4, pp. 88-91, Sep. 1963.
Stanley, I. G. Thayne, and D. R. S. Cumming, "A Planar Gunn Diode Operating Above $100 \mathrm{GHz}$," IEEE Electron Device Lett., vol. 28, no. 10, pp. 849-851, Oct. 2007.

[3] N. J. Pilgrim, A. Khalid, G. M. Dunn, and D. R. S. Cumming, "Gunn oscillations in planar heterostructure diodes," Semicond. Sci. Technol., vol. 23, no. 7, pp. 1-10, Jul. 2008.

[4] A. Khalid, C. Li, V. Papageorgiou, G. M. Dunn, M. J. Steer, I. G Thayne, M. Kuball, C. H. Oxley, M. Montes Bajo, A. Stephen, J. Glover, and D. R. S. Cumming, "In0.53Ga0.47As Planar Gunn Diodes Operating at a Fundamental Frequency of $164 \mathrm{GHz}$," IEEE Electron Device Lett., vol. 34, no. 1,pp.3941,jan.2013.[Online].http://ieeexplore.ieee.org/lpdocs/epic03/wr apper.htm?arnumber $=6352833$

[5] A. Khalid, C. Li, V. Papageorgiou, N. J. Pilgrim, G. M. Dunn, and D. R. S. Cumming, "A 218-GHz second-harmonic multi-quantum well GaAs-based planar Gunn diodes," Microw. Opt. Technol. Lett., vol. 55, no. 3, pp. 686-688, mar 2013. [Online]. Available: http://doi.wiley.com/10.1002/mop.27393

http://eprints.gla.ac.uk/86366/

[6] M. I. Maricar, PhD "Design of circuits to enhance the performance of high frequency planar Gunn diodes," De Montfort University, 2014.

[7] A. C. Kak, R. L. Gunshor, and C. P. Jethwa, "Equivalent-circuit representation for stably propagating domains in bulk GaAs," Electron. Lett., vol. 6, no. 22, p. 711, 1970

[8] C. $\mathrm{Li}, \mathrm{PhD}$ "Design and Characterisation of millimeter-wave planar Gunn diode and integrated circuits," University of Glasgow, 2011

[9] M. I. Maricar, A. Khalid, G. Dunn, and D. Cumming, "Experimentally estimated dead space for GaAs and InP based planar Gunn diodes," Semicond. Sci. Technol., vol. 30, p. 12001.

[10] J E Caroll, Hot electron microwave generators. Edward Arnold Ltd, London, 1970 